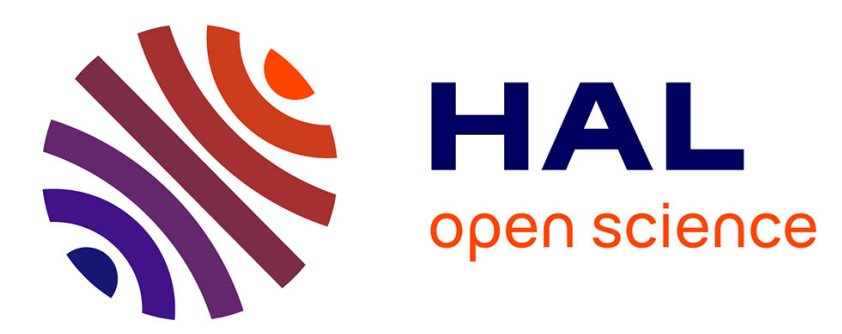

\title{
MCMC sampling for joint estimation of phase distortions and transmitted symbols in OFDM systems
}

François Septier, Yves Delignon

\section{To cite this version:}

François Septier, Yves Delignon. MCMC sampling for joint estimation of phase distortions and transmitted symbols in OFDM systems. Digital Signal Processing, 2011, 21 (2), pp.341-353. 10.1016/j.dsp.2010.10.003 . hal-00566491

\section{HAL Id: hal-00566491 https://hal-imt.archives-ouvertes.fr/hal-00566491}

Submitted on 16 Feb 2011

HAL is a multi-disciplinary open access archive for the deposit and dissemination of scientific research documents, whether they are published or not. The documents may come from teaching and research institutions in France or abroad, or from public or private research centers.
L'archive ouverte pluridisciplinaire $\mathbf{H A L}$, est destinée au dépôt et à la diffusion de documents scientifiques de niveau recherche, publiés ou non, émanant des établissements d'enseignement et de recherche français ou étrangers, des laboratoires publics ou privés. 


\title{
MCMC Sampling for Joint Estimation of Phase Distortions and Transmitted Symbols in OFDM Systems
}

\author{
François Septier*, Yves Delignon ${ }^{\dagger}$ \\ *Institut TELECOM/TELECOM Lille1 \\ 59658 Villeneuve d'Ascq, France \\ Email: francois.septier@telecom-lille1.eu \\ Telephone: (+ 33) 320436402 \\ $\dagger$ Institut TELECOM/TELECOM Lille1/ LAGIS FRE CNRS 3303 \\ 59658 Villeneuve d'Ascq, France \\ Email: yves.delignon@telecom-lille1.eu \\ Telephone: (+ 33) 320335570
}

\begin{abstract}
In this paper, we address the challenging problem of the OFDM reception in the presence of phase distortions. Phase noise and carrier frequency offset seriously degrade the performances of OFDM systems by destroying orthogonality of the subcarriers. Based on a Markov Chain Monte Carlo sampling mechanization, our approach consists in jointly estimating the phase noise, the frequency offset and the transmitted symbols. The proposed algorithm is implemented in the time domain in order to benefit from the redundancy information induced by the cyclic prefix and from the time correlation of the OFDM signal owing to the presence of virtual and/or pilot subcarriers. The algorithm's efficiency is enhanced by incorporating the Rao-Blackwellization technique as well as various sampling improvement strategies. Simulation results, provided in terms of bit error rate (BER) and mean square error (MSE), clearly illustrate the efficiency and the robustness of the proposed estimator.
\end{abstract}

\section{Index Terms}

Phase Noise, Carrier frequency offset, OFDM Systems, Markov Chain Monte Carlo. 


\section{INTRODUCTION}

Multicarrier modulation has been selected as the transmission scheme for the majority of communication systems such as the "Digital Subscriber lines" (DSL), the "Digital Audio Broadcast" (DAB), the IEEE 802.11 "Wireless Local Area Network" (WLAN), or the IEEE 802.16 "Wireless Metropolitan Area Network" (WMAN). Unfortunatly, such techniques are very sensitive to phase noise (PHN) and carrier frequency offset (CFO) caused by oscillators' impairments. These phase distortions destroy the subcarriers' orthogonality and lead after the Fourier Transform to rotation of every subcarriers, known as common phase error (CPE), and to inter-carrier interferences (ICI).

Many approaches have been proposed to estimate and eliminate phase noise effects in OFDM systems over frequency selective channels. Theses techniques can be divided into frequency domain [1]-[7] and time domain approaches [8]-[11]. For both of them, two different strategies based either on pilot or non-pilot aided algorithms have been employed. The latters are a challenging task since they have the advantage of being bandwidth more efficient as it does not require the transmission of pilots symbols. The frequency domain approaches in [5], [6] consist in correcting both CPE and ICI, induced by phase noise only, in the received signal obtained after the Fourier transform using pilot subcarriers. In a first step, CPE estimation is carried out with either a Kalman filter or a minimum mean square error pilot aided algorithm. Secondly, a decision feedback algorithm, using the demodulated symbols obtained after the CPE correction, is performed to obtain ICI estimate. However, noise-induced symbol decision errors may propagate through the feedback loop, leading to unreliable transmissions with error bursts. In [7], unlike the previous algorithms, the proposed scheme does not require the presence of pilot subcarriers. A decision-directed scheme is used at the initialization step in order to make a tentative decision on a prespecified set of subcarriers without any phase distortion correction. To have reliable tentative decision for the CPE estimation, slow phase noise variations and a residual CFO are considered.

In the time-domain approaches [9], [10], an approximate probabilistic inference technique associated with the minimization of variational free energy is proposed to jointly estimate phase noise and transmitted symbols without any pilot subcarriers. However, in order to derive the algorithm, the phase distortions term is linearized by the assumption of small phase noise rate (i.e. $e^{j \theta(t)} \approx 1+j \theta(t)$ ). Moreover, like in [7], a tentative symbol decision without any phase correction is made at initialization step and thus leads to a severe performance degradation when phase distortions are significant, especially in the presence of CFO (which is not considered in [9], [10]). Finally, a nonpilot-aided Sequential Monte Carlo method has been recently proposed in [11] to address this problem. 
In this paper, we propose a novel time domain approach for the joint estimation of the phase noise, CFO and transmitted symbols. The time-domain processing allows us to benefit from the redundancy information induced by the cyclic prefix and from the time correlation of the OFDM signal owing to the presence of virtual and/or pilot subcarriers. To solve this problem, the proposed algorithm is based on the Markov Chain Monte Carlo (MCMC) methodology, which have played a significant role in statistics, econometrics, physics and computing science over the last two decades [12]. Given the high-dimensional system, the algorithm's efficiency is enhanced by using the Rao-Blackwellization technique as well as powerful proposal distributions that faster drives new candidate samples towards high probability regions.

The paper is organized as follows. Section II is devoted to the problem formulation by introducing the OFDM system and the phase distortions. The proposed inference algorithm is detailed in Section III. Numerical results are shown in Section IV. Through different scenarios, our approach is compared to algorithms proposed in [5], [6], [11] and to a perfect CPE correction scheme.

In this paper, $N, N_{c p}$ and $T$ denote respectively the number of subcarriers, the cyclic prefix length and the OFDM symbol duration excluding the cyclic prefix. Let $\mathcal{N}(x ; \mu, \boldsymbol{\Sigma})$ and $\mathcal{N}_{c}(x ; \mu, \boldsymbol{\Sigma})$ represent respectively the real and circularly symmetric complex Gaussian random vectors with mean $\mu$ and covariance matrix $\boldsymbol{\Sigma} . \mathbf{I}_{n}, \mathbf{0}_{n \times m}$ and $\operatorname{diag}(\mathbf{x})$ are respectively the $n \times n$ identity matrix and the $n \times m$ matrix of zeros and a diagonal matrix with the vector $\mathrm{x}$ on its diagonal. Finally, lower case bold letters are used for column vectors and capital bold letters for matrices; $(.)^{*},(.)^{T}$ and $(.)^{H}$ denote respectively conjugate, transpose, and Hermitian transpose.

\section{PROBlEM Formulation}

\section{A. OFDM System model}

First, input independent and identically distributed (i.i.d.) bits are encoded into M-QAM symbols $d_{n, i}$, where $i$ denotes the $i^{\text {th }}$ subcarrier, $n$ the $n^{\text {th }}$ OFDM symbol. Practical OFDM systems are generally not fully loaded in order to avoid aliasing. In this case, some of the subcarriers at the edges of the multicarrier block are not modulated. These $N_{g}$ subcarriers are referred to as virtual subcarriers (VSCs). This number is dictated by system design requirements and is, in general, about $10 \%$ of subcarriers. We denote by $\Omega_{g}$ the set of virtual subcarriers. Moreover, to stay in a general multicarrier transmission, let us introduce a set of $N_{P}$ pilot subcarriers located on $\Omega_{p}$, with $\left\{\Omega_{p} \cap \Omega_{g}\right\}=\emptyset$ and $\Omega_{p} \cup \Omega_{g}=\Omega$. These considerations 
can be summed up as :

$$
d_{n, i}= \begin{cases}\text { Information Data } & i \notin \Omega \\ \text { Pilot } & i \in \Omega_{p} \\ 0 & i \in \Omega_{g}\end{cases}
$$

After the Inverse Discrete Fourier Tranform (IDFT), the samples of the transmitted signal can be written, for $l=0 . . N-1$, as :

$$
s_{n, l}=\frac{1}{\sqrt{N}} \sum_{i=0}^{N-1} d_{n, i} e^{j 2 \pi i l / N}
$$

By considering the presence of virtual or pilot subcarriers, the transmitted signal $s_{n, l}$ is decomposed into a known signal $\kappa_{n, l}$ and an unknown signal $u_{n, l}$ :

$$
s_{n, l}=\kappa_{n, l}+u_{n, l}
$$

with, using (1) and (2),

$$
\kappa_{n, l}=\frac{1}{\sqrt{N}} \sum_{i \in \Omega} d_{n, i} e^{j 2 \pi i l / N}
$$

and

$$
u_{n, l}=\frac{1}{\sqrt{N}} \sum_{\substack{i=0 \\ \notin \Omega}}^{N-1} d_{n, i} e^{j 2 \pi i l / N}
$$

As a consequence, $\mathbf{u}_{n}=\left[u_{n, N-1}, \ldots, u_{n, 0}\right]^{T}$ represents the signal of interest. Based on the central limit theorem, a rigorous proof given in [13] establishes that the complex envelope of a bandlimited uncoded OFDM signal converges to a Gaussian random process:

$$
p\left(\mathbf{u}_{n}\right)=\mathcal{N}\left(\mathbf{u}_{n} \mid \mathbf{0}_{N \times 1}, \mathbf{C}_{u}\right)
$$

where the covariance matrix is defined as :

$$
\mathbf{C}_{u}=\left[\begin{array}{cccc}
C_{u}(0) & C_{u}(1) & \cdots & C_{u}(N-1) \\
C_{u}(-1) & C_{u}(0) & \cdots & C_{u}(N-2) \\
\vdots & \vdots & \ddots & \vdots \\
C_{u}(-N+1) & C_{u}(-N+2) & \cdots & C_{u}(0)
\end{array}\right]
$$

The correlation between discrete-time samples of the unknown signal is obtained using the definition of $u_{n, l}$ in (5) and $\mathbb{E}\left[d_{n, i} d_{n, i^{\prime}}^{*}\right]=\delta\left(i-i^{\prime}\right)$ :

$$
\begin{aligned}
C_{u}(l) & \triangleq \mathbb{E}\left[u_{n, t} u_{n, t-l}^{*}\right]=\frac{1}{N} \sum_{\substack{i=0 \\
\notin \Omega}}^{N-1} \sum_{\substack{i^{\prime}=0 \\
\notin \Omega}}^{N-1} \mathbb{E}\left[d_{n, i} d_{n, i^{\prime}}^{*}\right] e^{j 2 \pi i t / N} e^{-j 2 \pi i^{\prime}(t-l) / N} \\
& =\frac{1}{N} \sum_{\substack{i=0 \\
\notin \Omega}}^{N-1} e^{j 2 \pi i l / N}
\end{aligned}
$$


Before transmission, a cyclic prefix (CP) of length $N_{c p}$ is introduced to remove inter-symbol interference (ISI). The resulting signal (Fig. 1) is transmitted in a time varying frequency selective channel $h(t, \tau)$ which is assumed to be static over one OFDM symbol and characterized by $L_{p}$ independent propagation paths. Assuming perfect frequency, phase and timing synchronization, the $n^{\text {th }}$ received OFDM symbol can be expressed, in a matrix form, as :

$$
\begin{aligned}
\mathbf{r}_{n} & =\mathbf{H}_{n} \mathbf{A} \mathbf{s}_{n}+\boldsymbol{\nu}_{n}+\mathbf{b}_{n} \\
& =\mathbf{H}_{n} \mathbf{A}\left(\boldsymbol{\kappa}_{n}+\mathbf{u}_{n}\right)+\boldsymbol{\nu}_{n}+\mathbf{b}_{n}
\end{aligned}
$$

where $\mathbf{r}_{n}=\left[r_{n, N+N_{c p}-1}, \ldots, r_{n, 0}\right]^{T}, \mathbf{s}_{n}=\left[s_{n, N-1}, \ldots, s_{n, 0}\right]^{T}, \boldsymbol{\kappa}_{n}=\left[\kappa_{n, N-1}, \ldots, \kappa_{n, 0}\right]^{T}, \mathbf{u}_{n}=\left[u_{n, N-1}, \ldots, u_{n, 0}\right]^{T}$ and $\mathbf{b}_{n}=\left[b_{n, N+N_{c p}-1}, \ldots, b_{n, 0}\right]^{T}$ correspond to the received signal, the transmitted signal, the known and unknown component of the transmitted signal and the additive white Gaussian noise with covariance matrix $\sigma_{b}^{2} \mathbf{I}_{N+N_{c p}}$, respectively. The matrices $\mathbf{H}_{n}$ of dimension $\left(N+N_{c p}\right) \times\left(N+N_{c p}\right)$ and $\mathbf{A}$ are defined as follows :

$$
\mathbf{H}_{n}=\left[\begin{array}{ccccccc}
h_{n, 0} & h_{n, 1} & \cdots & h_{n, L_{p}-1} & 0 & \cdots & 0 \\
0 & h_{n, 0} & h_{n, 1} & \cdots & h_{n, L_{p}-1} & \ddots & \vdots \\
\vdots & \ddots & \ddots & & & \ddots & 0 \\
0 & \cdots & \cdots & \cdots & \cdots & 0 & h_{n, 0}
\end{array}\right]
$$

and

$$
\mathbf{A}=\left[\begin{array}{c}
\mathbf{I}_{N} \\
\mathbf{I}_{N_{c p}} \quad \mathbf{0}_{N_{c p} \times\left(N-N_{c p}\right)}
\end{array}\right]
$$

Finally, $\boldsymbol{\nu}_{n}$ represents the ISI between two consecutives OFDM symbols caused by the multipath channel

$$
\boldsymbol{\nu}_{n}=\left[\begin{array}{c}
\mathbf{0}_{N+N_{c p}-L_{p}+1 \times 1} \\
\sum_{p=L_{p}-2}^{L_{p}-1} h_{n, p} s_{n-1, N+L_{p}-p-3} \\
\vdots \\
\sum_{p=1}^{L_{p}-1} h_{n, p} s_{n-1, N+L_{p}-p-3}
\end{array}\right]
$$

Then by assuming $N_{c p} \geqslant L_{p}$ and after discarding the cyclic prefix, a fast Fourier transform is applied on the remaining $N$ samples (i.e. $\left\{r_{n, k}\right\}_{k=N_{c p}}^{N+N_{c p}-1}$ ) in order to recover the desired data using an equalization technique. However, analog front-end impairments, such as noise, distortion and mismatch, may affect performance by degrading the integrity of the desired signal. In this paper, we focus on phase noise (PHN) and carrier frequency offset (CFO) caused by oscillator instabilities. 


\begin{tabular}{|c|c|c|c|c|c|c|c|c|c|c|c|}
\hline & & \multicolumn{8}{|c|}{$n^{\text {th }}$ OFDM symbol } & & \\
\hline . & $s_{n-1, N-1}$ & $s_{n, N-N_{c p}}$ & $s_{n, N-1}$ & $s_{n, 0}$ & $s_{n, 1}$ & $\cdots$ & $s_{n, N-N_{c p}}$ & $\cdots$ & $s_{n, N-1}$ & $s_{n+1, N-N_{c p}}$ & $\cdots$ \\
\hline
\end{tabular}

Fig. 1. Example of an OFDM flow.

\section{B. Phase Distortion}

Imperfect oscillators at the transmitter and at the receiver introduce a phase noise and a carrier frequency offset $\Delta f$ so that, at the sampling rate $N / T$ of the receiver, the discrete form of the carrier delivered by the noisy oscillator on the $k^{\text {th }}$ sample of the $n^{\text {th }}$ OFDM symbol is :

$$
p_{n, k}=\exp \left(j \theta_{n, k}+\frac{2 \pi k \epsilon}{N}\right) \quad \forall k=0, \cdots, N+N_{c p}-1
$$

where $\theta_{n, k}$ and $\epsilon$ correspond respectively to the phase noise (PHN) and the normalized CFO. In this work, we assume that $\epsilon$ is uniformly drawn on $\left[-\Delta_{\mathrm{CFO}} ; \Delta_{\mathrm{CFO}}\right]$ :

$$
p(\epsilon)=\mathcal{U}\left(\epsilon \mid-\Delta_{\mathrm{CFO}}, \Delta_{\mathrm{CFO}}\right)
$$

The discrete-time samples of the PHN process form a random-walk process $\theta_{n, k}=\theta_{n, k-1}+w_{n, k}$, $k=0, \ldots, N+N_{c p}-1$, where $w_{n, t}$ is a white Gaussian noise with variance $\sigma_{w}^{2}=2 \pi \beta \frac{T}{N}$. $\beta$ is the bandwidth of the Brownian phase noise normalized with respect to the OFDM symbol rate $\frac{1}{T}$, namely the parameter $\beta T$. If a perfect phase synchronization at the beginning of the OFDM symbol (i.e. $\theta_{n,-1}=0$ ) is assumed as in [10], [14], the prior distribution of the PHN vector $\boldsymbol{\theta}_{n}=\left[\theta_{n, N+N_{c p}-1}, \ldots, \theta_{n, 0}\right]^{T}$ is given by:

$$
p\left(\boldsymbol{\theta}_{n}\right)=\mathcal{N}\left(\boldsymbol{\theta}_{n} \mid \mathbf{0}_{N+N_{c p} \times 1}, \boldsymbol{\Theta}\right)
$$

with

$$
\Theta=\frac{2 \pi \beta T}{N}\left[\begin{array}{cccc}
N+N_{c p} & & 2 & 1 \\
& \ddots & \vdots & \vdots \\
2 & \cdots & 2 & 1 \\
1 & \cdots & 1 & 1
\end{array}\right]
$$

The received signal $\mathbf{r}_{n}$ in (9) corrupted by both phase noise and frequency offset becomes :

$$
\mathbf{r}_{n}=\boldsymbol{\Phi}_{n}\left(\mathbf{H}_{n} \mathbf{A}\left(\boldsymbol{\kappa}_{n}+\mathbf{u}_{n}\right)+\boldsymbol{\nu}_{n}\right)+\mathbf{b}_{n}
$$


where $\boldsymbol{\Phi}_{n}=\operatorname{diag}\left(e^{j\left(\boldsymbol{\theta}_{n}+\mathbf{g} \epsilon\right)}\right)$ with $\mathbf{g}=\left[2 \pi\left(N+N_{c p}-1\right) / N, \ldots, 2 \pi / N, 0\right]^{T}$.

If no correction method is employed, these phase distortions lead, after the discrete Fourier transform, to two types of distortions on the useful signal, a multiplicative one which is common to all subcarriers and known as Common Phase Error (CPE) and an additive inter-carrier interference (ICI). Larger the phase noise rate $\beta T$ and the $\mathrm{CFO}$ support $\Delta_{\mathrm{CFO}}$ are, the worse the SINR (signal to noise plus interference ratio) is. Furthermore, on a same bandwidth a larger number of subcarriers leads to worse system performance due to the shorter subcarrier spacing distance for a same two-sided $3 \mathrm{~dB}$ bandwith of the phase noise [4]. In order to have a reliable multicarrier system, a phase distortion compensation scheme is consequently required.

\section{BAYESIAN SOLUTION}

In a Bayesian context, the aim is to compute the posterior distribution $p\left(\mathbf{u}_{n}, \boldsymbol{\theta}_{n}, \epsilon \mid \mathbf{r}_{n}\right)$. However, this complex and highly nonlinear distribution cannot be obtained analytically and approximations should be made instead. We propose to approximate it by using a Markov Chain Monte Carlo (MCMC) algorithm. Moreover, in order to improve the accuracy of the algorithm, we propose to apply the RaoBlackwellization principle to the unknown OFDM signal $\mathbf{u}_{n}$.

\section{A. Some Generalities about MCMC methods}

The key idea of MCMC methods is to generate samples by running an ergodic chain whose equilibrium distribution is the desired distribution [12], [15], [16]. An aperiodic and irreducible Markov chain has the property of converging to a unique stationary distribution, $\pi(\mathbf{x})$, which does not depend on the initial sample or the iteration number $m$. After convergence, a MCMC method produces samples from the target distribution, $\pi(\mathbf{x})$, so that :

$$
\pi(\mathbf{x}) \approx \frac{1}{M} \sum_{m=1}^{M} \delta\left(\mathbf{x}-\mathbf{x}^{m}\right)
$$

The Metropolis-Hastings (MH) algorithm, summarized in Algorithm 1, is the most popular MCMC method [17]. We should remark that it is standard practice to discard a number of initial "burn-in" samples, denoted here by $B$, to allow the MCMC algorithm to converge to the stationary distribution. The MH algorithm is very simple, but it requires careful design of the proposal ditribution $q\left(\mathbf{x} \mid \mathbf{x}^{m-1}\right)$, especially when the dimension of the state $\mathrm{x}$ is large. Another well-known MCMC method is the Gibbs sampler [18]. This scheme is a special case of the $\mathrm{MH}$ algorithm that generates samples from conditional densities which make the probability of acceptance equal to one. The Gibbs sampling method is generally more 
effective than the MH algorithm in high-dimensional systems. When the full conditionals are available and belong to the family of standard distributions (Gamma, Gaussian, etc.), samples can be sampled directly from them. Otherwise, we can draw samples with MH steps embedded within the Gibbs algorithm.

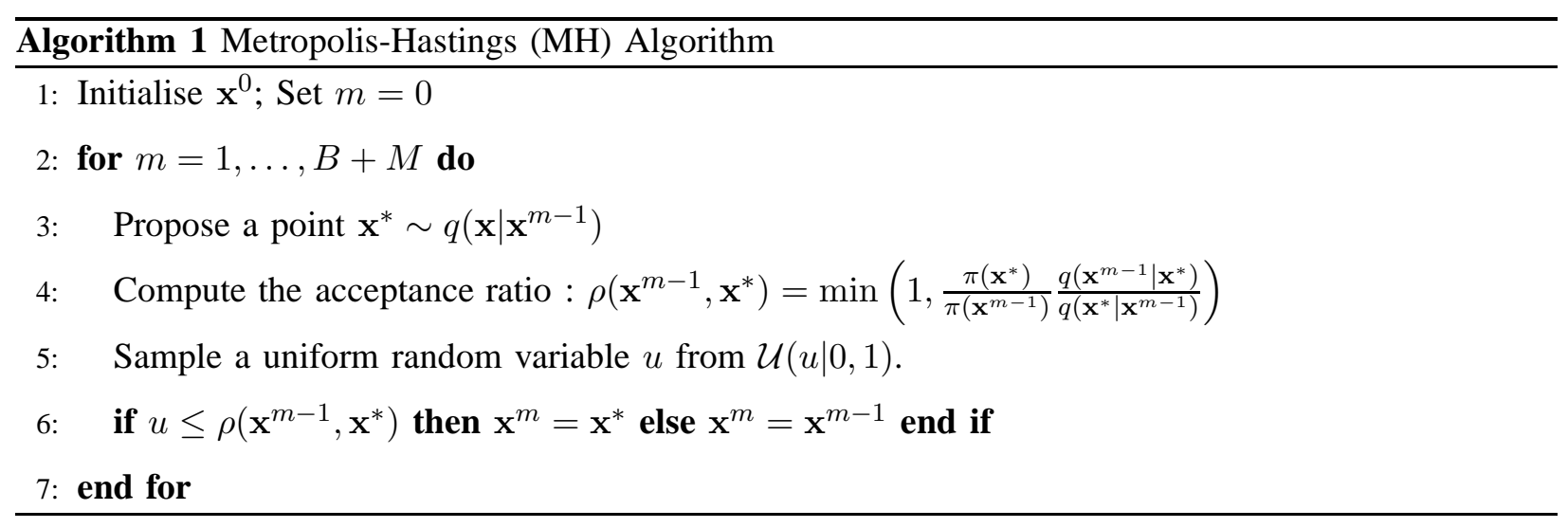

\section{B. Rao-Blackwellization Technique}

In this work the target distribution is the posterior distribution, $p\left(\mathbf{u}_{n}, \boldsymbol{\theta}_{n}, \epsilon \mid \mathbf{r}_{n}\right)$. Given the high dimensionality of the state space, it is not straightforward to design an efficient MCMC algorithm. However, by using the linear and Gaussian sub-structure of the system, the state space can be reduced significantly by marginalizing out the unknown OFDM signal $\mathbf{u}_{n}$. Indeed, using the Bayes' theorem, the posterior distribution of interest can be expanded as :

$$
p\left(\mathbf{u}_{n}, \boldsymbol{\theta}_{n}, \epsilon \mid \mathbf{r}_{n}\right)=p\left(\mathbf{u}_{n} \mid \mathbf{r}_{n}, \boldsymbol{\theta}_{n}, \epsilon\right) p\left(\boldsymbol{\theta}_{n}, \epsilon \mid \mathbf{r}_{n}\right)
$$

Since both the prior distribution of $\mathbf{u}_{n}$, defined in (6), and the likelihood distribution, obtained using (17), are Gaussian, it is straightforward to show that

$$
p\left(\mathbf{u}_{n} \mid \mathbf{r}_{n}, \boldsymbol{\theta}_{n}, \epsilon\right)=\mathcal{N}_{c}\left(\mathbf{u}_{n} \mid \widehat{\mathbf{u}}_{n}, \boldsymbol{\Sigma}_{n}\right)
$$

with,

$$
\widehat{\mathbf{u}}_{n}=\mathbf{C}_{u} \boldsymbol{\Lambda}_{n}^{H}\left[\boldsymbol{\Lambda}_{n} \mathbf{C}_{u} \boldsymbol{\Lambda}_{n}^{H}+\sigma_{b}^{2} \mathbf{I}_{N+N_{c p}}\right]^{-1}\left(\mathbf{r}_{n}-\boldsymbol{\Lambda}_{n} \boldsymbol{\kappa}_{n}-\boldsymbol{\Phi}_{n} \boldsymbol{\nu}_{n}\right)
$$

and

$$
\boldsymbol{\Sigma}_{n}=\left(\mathbf{I}_{N}-\mathbf{C}_{u} \boldsymbol{\Lambda}_{n}^{H}\left[\boldsymbol{\Lambda}_{n} \mathbf{C}_{u} \boldsymbol{\Lambda}_{n}^{H}+\sigma_{b}^{2} \mathbf{I}_{N+N_{c p}}\right]^{-1} \boldsymbol{\Lambda}_{n}\right) \mathbf{C}_{u}
$$

where $\boldsymbol{\Lambda}_{n}=\boldsymbol{\Phi}_{n} \mathbf{H}_{n} \mathbf{A}$. 
Using the Rao-Blackwellization principle on $\mathbf{u}_{n}$, only $p\left(\boldsymbol{\theta}_{n}, \epsilon \mid \mathbf{r}_{n}\right)$ needs to be approximated using a MCMC method. The posterior distribution (19) will therefore be approximated by :

$$
p\left(\mathbf{u}_{n}, \boldsymbol{\theta}_{n}, \epsilon \mid \mathbf{r}_{n}\right) \approx \frac{1}{M} \sum_{m=1}^{M} p\left(\mathbf{u}_{n} \mid \mathbf{r}_{n}, \boldsymbol{\theta}_{n}^{m}, \epsilon^{m}\right) \delta\left(\boldsymbol{\theta}_{n}-\boldsymbol{\theta}_{n}^{m} ; \epsilon-\epsilon^{m}\right)
$$

where $\left\{\boldsymbol{\theta}_{n}^{m}, \epsilon^{m}\right\}_{m=1}^{M}$ represents the converged samples of the MCMC algorithm.

\section{Proposed MCMC method}

In this paper, we propose to design a MCMC for approximating $p\left(\boldsymbol{\theta}_{n}, \epsilon \mid \mathbf{r}_{n}\right)$ which can be written, using the Bayes' theorem, as:

$$
p\left(\boldsymbol{\theta}_{n}, \epsilon \mid \mathbf{r}_{n}\right) \propto p\left(\mathbf{r}_{n} \mid \boldsymbol{\theta}_{n}, \epsilon\right) p\left(\boldsymbol{\theta}_{n}\right) p(\epsilon)
$$

The prior distribution of $\boldsymbol{\theta}_{n}$ and $\epsilon$ are defined in (15) and (14), respectively. The likelihood distribution is given, using (17) and (6), by :

$$
\begin{aligned}
p\left(\mathbf{r}_{n} \mid \boldsymbol{\theta}_{n}, \epsilon\right) & =\int p\left(\mathbf{r}_{n} \mid \mathbf{u}_{n}, \boldsymbol{\theta}_{n}, \epsilon\right) p\left(\mathbf{u}_{n}\right) d \mathbf{u}_{n} \\
& =\mathcal{N}_{c}\left(\mathbf{r}_{n} \mid \boldsymbol{\Lambda}_{n} \boldsymbol{\kappa}_{n}+\boldsymbol{\Phi}_{n} \boldsymbol{\nu}_{n}, \boldsymbol{\Lambda}_{n} \mathbf{C}_{u} \boldsymbol{\Lambda}_{n}^{H}+\sigma_{b}^{2} \mathbf{I}_{N+N_{c p}}\right)
\end{aligned}
$$

Careful design of the sampling strategy plays an important role in the success of a MCMC algorithm. Here, we propose to use a series of Metropolis-Hastings-within-Gibbs. More precisely, at the $m^{\text {th }}$ MCMC iteration, the following procedure is adopted to produce samples from $p\left(\boldsymbol{\theta}_{n}, \epsilon \mid \mathbf{r}_{n}\right)$ :

1) We first make a proposal for $\epsilon$ using a MH step.

2) Then, the sampling of $\boldsymbol{\theta}_{n}$ is performed by dividing the PHN vector in $I$ blocks, i.e. $\boldsymbol{\theta}_{n}=\left\{\boldsymbol{\theta}_{n, i}\right\}_{i=1}^{I}$, that are successively sampled using a series of MH steps.

Let us now describe in more details these two steps.

1) Proposal for the CFO: To carry out the draw of $\epsilon$, we propose to use a $\mathrm{MH}$ procedure in which the proposal distribution is given by :

$$
q\left(\epsilon \mid \epsilon^{m-1}\right)=P_{\epsilon} \mathcal{U}\left(\epsilon \mid-\Delta_{\mathrm{CFO}}, \Delta_{\mathrm{CFO}}\right)+\left(1-P_{\epsilon}\right) \mathcal{N}\left(\epsilon \mid \epsilon^{m-1}, \sigma_{\epsilon}^{2}\right)
$$

with $0 \leq P_{\epsilon} \leq 1$. This proposal is based on a mixture of the prior distribution of $\epsilon$ and a Gaussian distribution centered on the current Markov chain state, $\epsilon^{m-1}$, and with variance $\sigma_{\epsilon}^{2}$. The acceptance ratio is given by :

$$
\rho_{1}=\min \left(1, \frac{p\left(\mathbf{r}_{n} \mid \boldsymbol{\theta}_{n}^{m-1}, \epsilon^{*}\right) p\left(\epsilon^{*}\right)}{p\left(\mathbf{r}_{n} \mid \boldsymbol{\theta}_{n}^{m-1}, \epsilon^{m-1}\right) p\left(\epsilon^{m-1}\right)} \frac{q\left(\epsilon^{m-1} \mid \epsilon^{*}\right)}{q\left(\epsilon^{*} \mid \epsilon^{m-1}\right)}\right)
$$


2) Proposal for the PHN: Given the high-dimension of the PHN vector $\boldsymbol{\theta}_{n}$, we propose to divide this vector in $I$ blocks and then to successively sample them. The $i^{\text {th }}$ block is defined as $\boldsymbol{\theta}_{n, i}=$ $\left[\theta_{n, i \frac{N+N_{c p}}{I}-1}, \ldots, \theta_{n,(i-1) \frac{N+N_{c p}}{I}}\right]^{T}$ and $\boldsymbol{\theta}_{n, \backslash i}$ denotes the collection of $\left\{\theta_{n, k}\right\}_{k=0}^{N+N_{c p}}$ excluding $\boldsymbol{\theta}_{n, i}$. For sampling $\boldsymbol{\theta}_{n, i}$, we propose the following proposal distribution:

$$
q\left(\boldsymbol{\theta}_{n, i} \mid \boldsymbol{\theta}_{n}^{m}, \epsilon^{m}, \mathbf{r}_{n}\right)=P_{\theta} p\left(\boldsymbol{\theta}_{n, i} \mid \boldsymbol{\theta}_{n, \backslash i}^{m}\right)+\left(1-P_{\theta}\right) q_{1}\left(\boldsymbol{\theta}_{n, i} \mid \boldsymbol{\theta}_{n}^{m}, \epsilon^{m}, \mathbf{r}_{n}\right)
$$

with $0 \leq P_{\theta} \leq 1$. Like for the CFO, this proposal is a mixture of two distributions. With probability $P_{\theta}$, $\boldsymbol{\theta}_{n, i}^{*}$ is drawn from its conditional prior distribution $p\left(\boldsymbol{\theta}_{n, i} \mid \boldsymbol{\theta}_{n, \backslash i}^{m}\right)$. Since $p\left(\boldsymbol{\theta}_{n}\right)$ is a Gaussian distribution, this conditional distribution is also a Gaussian distribution :

$$
p\left(\boldsymbol{\theta}_{n, i} \mid \boldsymbol{\theta}_{n, i i}^{m}\right)=\mathcal{N}\left(\boldsymbol{\theta}_{n, i} \mid \overline{\boldsymbol{\theta}}_{n, i}^{m}, \overline{\boldsymbol{\Theta}}_{n, i}\right)
$$

where the mean vector and the covariance matrix are respectively given by $\overline{\boldsymbol{\theta}}_{n, i}^{m}=\boldsymbol{\Theta}_{i}^{12}\left(\boldsymbol{\Theta}_{i}^{22}\right)^{-1} \boldsymbol{\theta}_{n, \backslash i}^{m}$ and $\overline{\boldsymbol{\Theta}}_{n, i}=\boldsymbol{\Theta}_{i}^{11}-\boldsymbol{\Theta}_{i}^{12}\left(\boldsymbol{\Theta}_{i}^{22}\right)^{-1} \boldsymbol{\Theta}_{i}^{21}$ with $\boldsymbol{\Theta}_{i}^{11}=\mathbb{E}\left[\boldsymbol{\theta}_{n, i} \boldsymbol{\theta}_{n, i}^{T}\right], \boldsymbol{\Theta}_{i}^{12}=\mathbb{E}\left[\boldsymbol{\theta}_{n, i} \boldsymbol{\theta}_{n, i}^{T}\right], \boldsymbol{\Theta}_{i}^{21}=\mathbb{E}\left[\boldsymbol{\theta}_{n, i} \boldsymbol{\theta}_{n, i}^{T}\right]$ and $\boldsymbol{\Theta}_{i}^{22}=\mathbb{E}\left[\boldsymbol{\theta}_{n, \backslash i} \boldsymbol{\theta}_{n, \backslash i}^{T}\right]$ obtained from (15).

With probability $\left(1-P_{\theta}\right), \boldsymbol{\theta}_{n, i}^{*}$ is drawn from $q_{1}\left(\boldsymbol{\theta}_{n, i} \mid \boldsymbol{\theta}_{n}^{m}, \epsilon^{m}, \mathbf{r}_{n}\right)$ in which the observations are taken into account in order to improve the sampling process. As detailed in Appendix A, this proposal is defined as :

$$
q_{1}\left(\boldsymbol{\theta}_{n, i} \mid \boldsymbol{\theta}_{n}^{m}, \epsilon^{m}, \mathbf{r}_{n}\right)=\mathcal{N}\left(\boldsymbol{\theta}_{n, i} \mid \widehat{\boldsymbol{\theta}}_{n, i}^{m}, \boldsymbol{\Sigma}_{\theta, i}^{m}\right)
$$

where the mean vector and the covariance matrix are obtained using the linearization of $\exp \left(j \bar{\theta}_{n, i}\right)$ - Eqs. (37)-(41). This proposal allows to drive new candidates towards high probability regions. The acceptance ratio associated to the PHN sampling is given by :

$$
\rho_{2}=\min \left(1, \frac{p\left(\mathbf{r}_{n} \mid \boldsymbol{\theta}_{n, i}^{*}, \boldsymbol{\theta}_{n, \backslash i}^{m}, \epsilon^{m}\right) p\left(\boldsymbol{\theta}_{n, i}^{*}, \boldsymbol{\theta}_{n, \backslash i}^{m}\right)}{p\left(\mathbf{r}_{n} \mid \boldsymbol{\theta}_{n}^{m}, \epsilon^{m}\right) p\left(\boldsymbol{\theta}_{n}^{m}\right)} \frac{q\left(\boldsymbol{\theta}_{n, i}^{m} \mid \boldsymbol{\theta}_{n, \backslash i}^{m}, \boldsymbol{\theta}_{n, i}^{*}, \epsilon^{m}, \mathbf{r}_{n}\right)}{q\left(\boldsymbol{\theta}_{n, i}^{*} \mid \boldsymbol{\theta}_{n}^{m}, \epsilon^{m}, \mathbf{r}_{n}\right)}\right)
$$

The proposed MCMC algorithm for the joint estimation of the OFDM signal, the phase noise and the carrier frequency offset, denoted by JSPCE-MCMC, is summarized in Algorithm 2.

\section{Results}

In order to show the validity of our approach, extensive simulations have been performed. In a first part, the case of PHN without CFO is considered in order to study the joint PHN and OFDM signal estimation. Then in the last part, the performances of the JSPCE-MCMC are assessed when both CFO and PHN are present in OFDM systems. In these two different cases, JSPCE-MCMC is studied in terms 


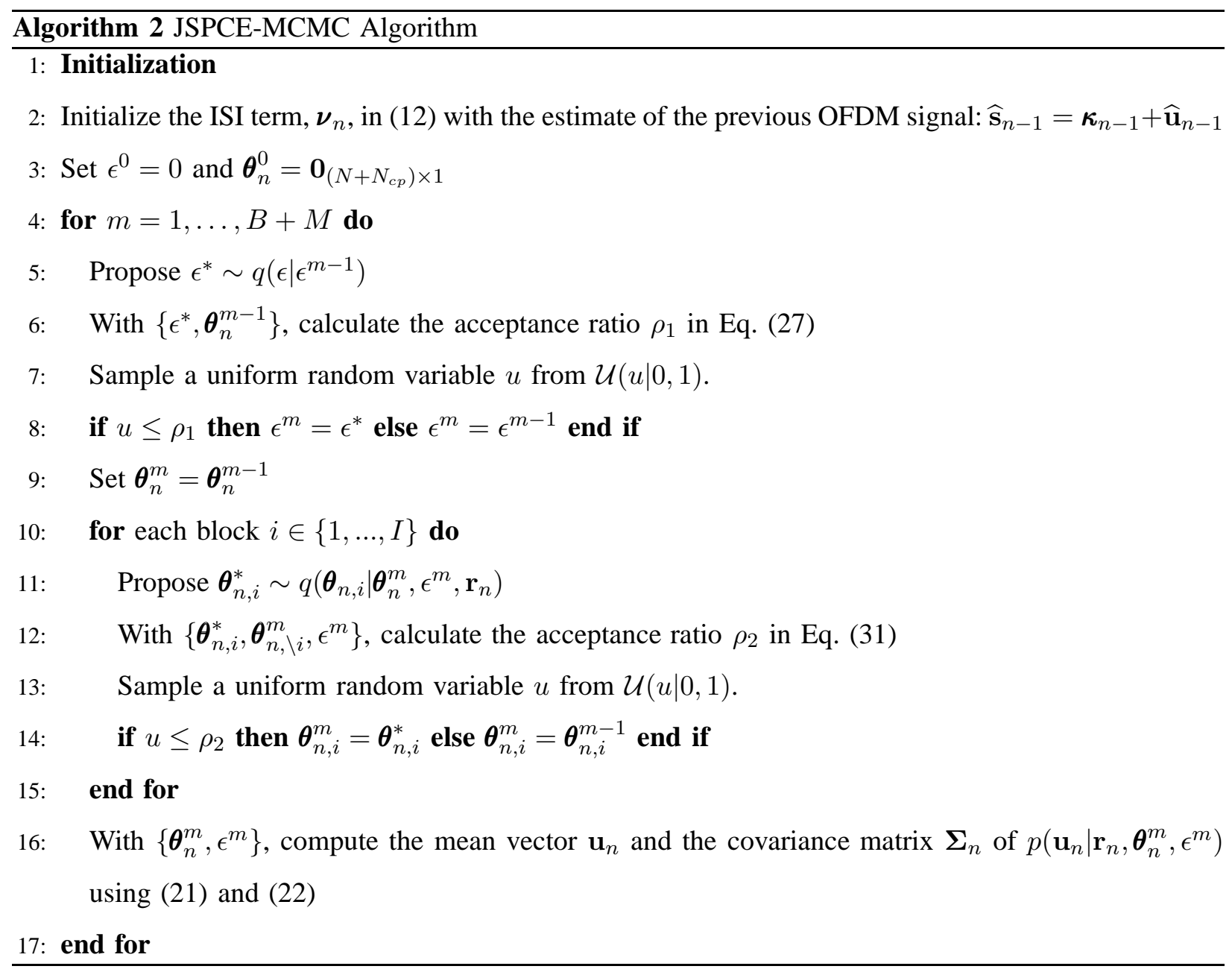

of mean square error (MSE) and bit error rate (BER) and compared to existing methods. Moreover, the BER performance of an OFDM system in the absence of phase distortions and using the classical frequency domain MMSE equalizer is also depicted and denoted below by MMSE-FEQ.

With regard to the system parameters, 16-QAM modulation is assumed and we have chosen $N=64$ subcarriers with a cyclic prefix of length $N_{c p}=8$. A Rayleigh frequency selective channel with $L=4$ paths and an uniform power delay profile, perfectly known by the receiver, has been generated for each OFDM symbol. The proposed JSPCE-MCMC is implemented with 200 MCMC iterations and a burn-in of 100 iterations. The particle approximation of $p\left(\mathbf{u}_{n}, \boldsymbol{\theta}_{n}, \epsilon \mid \mathbf{r}_{n}\right)$ is therefore obtained by using the MCMC samples after the intial burn-in iterations. 


\section{A. Performances with PHN only (i.e. $\epsilon=0$ )}

We first perform simulations with no CFO in order to study the joint PHN and multicarrier signal estimation. Performances of proposed algorithm are analyzed and compared to the CPE and ICI correction method proposed in [5], [6] and also to a perfect CPE correction scheme. From figure 2, it can be clearly seen that the JSPCE-MCMC outperforms the CPE+ICI correction scheme. In a phase distortion-free context and by excluding the cyclic prefix in the received signal, the proposed algorithm leads to a timedomain MMSE equalizer since only Eq. (20) is used to obtain an estimate of the OFDM signal. Time domain and frequency domain MMSE equalization are mathematically equivalent and result in the same performance [19]. Consequently, the performance gain between the MMSE-FEQ and the JSPCE-MCMC without distortions clearly highlights the benefit of considering the additional information induced by the cyclic prefix as well as the time-correlation of the OFDM signal owing to the presence of virtual and/or pilot subcarriers.

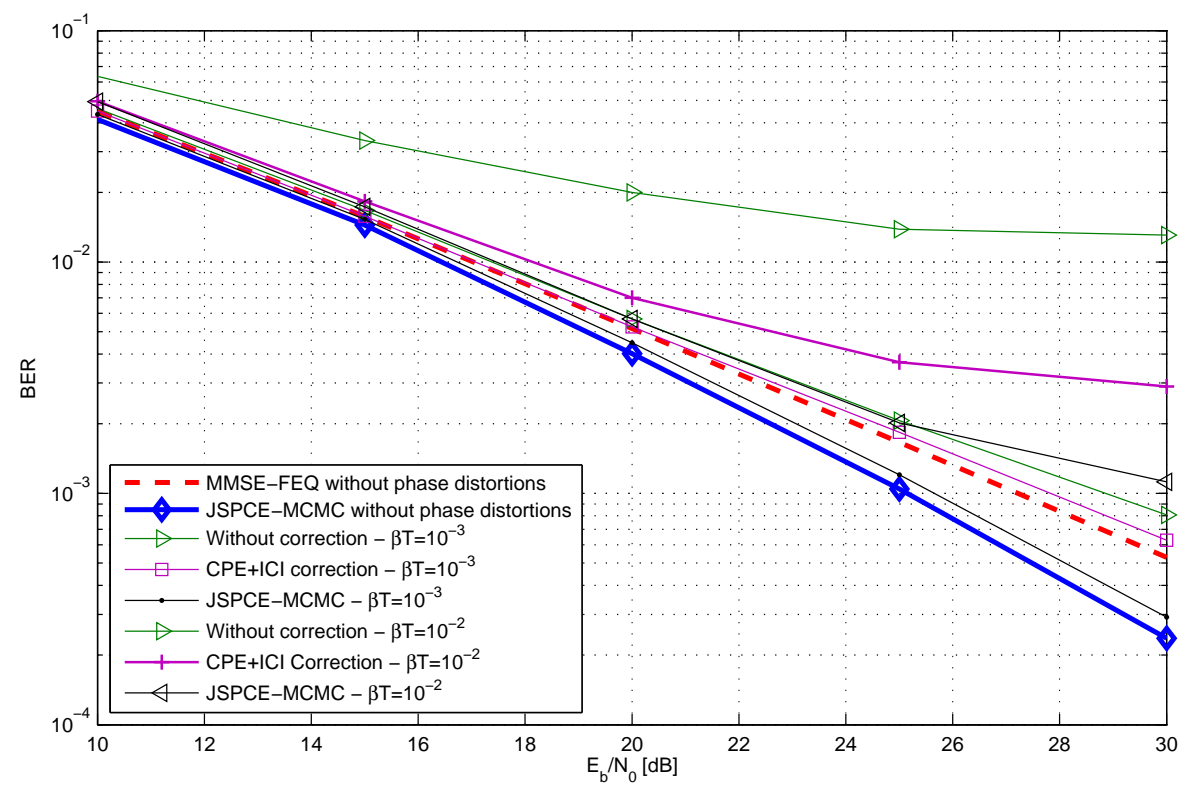

Fig. 2. BER performance of the proposed JSPCE-MCMC vs $E_{b} / N_{0}$ for different PHN rates $\beta T$ in an OFDM system $\left(N_{g}=0\right.$, $P=4, \epsilon=0)$.

Fig. 3 shows the impact of the number of null-subcarriers on JSPCE-MCMC performance in term of BER. From this figure, it can be denoted that its performance increases with the number of nullsubcarriers, especially for large PHN rate. Indeed, if the number of pilots or null-subcarriers increases, 
the proposed model of the multicarrier signal gives more prior information due to the correlation of time domain samples in $\mathbf{C}_{u}$ - Eq. (6). As a consequence, this leads to an improvement of the estimator robustness against high phase distortions level. In order to illustrate this gain, the MSE of both multicarrier signal and phase distortions obtained using the JSPCE-MCMC with and without the correlation of the OFDM time domain samples is shown in Figs. 4 and 5. For the latter configuration, the JSPCE-MCMC is performed by using in (6) the following covariance :

$$
\mathbf{C}_{u}=\left[\begin{array}{cccc}
C_{u}(0) & 0 & \cdots & 0 \\
0 & C_{u}(0) & \cdots & 0 \\
\vdots & \vdots & \ddots & \vdots \\
0 & \cdots & 0 & C_{u}(0)
\end{array}\right]
$$

From these two figures, it can be observed that the MSE of both multicarrier signal and phase distortions is significantly improved by taking into account the correlation between time domain samples of the OFDM signal, especially in severe PHN context. Indeed, for $E_{b} / N_{0}=30 \mathrm{~dB}$ and $\beta T=10^{-2}$, the MSE of the multicarrier signal obtained with the JSPCE-MCMC using the time correlation is $7 \times 10^{-3}$ and $4 \times 10^{-3}$ for respectively $N_{g}=0$ and $N_{g}=8$.

Most of the works, related in multicarrier receivers, assume that the duration of cyclic prefix is longer than the maximum delay spread of the multipath channel. Under this assumption, the system is free from ISI. Nevertheless, the addition of the cyclic prefix reduces the bandwidth utilization efficiency. It is intuitive that the duration of cyclic prefix should be kept as short as possible. As a result, the duration of cyclic prefix may be shorter than the maximum delay of the multipath channel in some occasional cases. In these cases, ISI and ICI exist and, hence deteriorate the performance of the system. Here, performances of the proposed JSPCE-MCMC algorithm are assessed with insufficient cyclic prefix and with $P=4$ pilots inserted in each OFDM symbol $\left(N_{g}=0\right)$.

Fig. 6 shows BER performance of the proposed scheme versus $E_{b} / N_{0}$ for two different number of channel paths and PHN rates. Whatever the PHN rate is, the JSPCE-MCMC significantly outperforms a classical OFDM receiver using MMSE equalizer without PHN. Since the proposed scheme is implemented in time-domain and has the advantage of taking into account the redundancy of the cyclic prefix, the JSPCE-MCMC is more robust against the ISI than the classical MMSE equalizer.

\section{B. Performances with both PHN and CFO}

In the following simulations, the CFO term $\epsilon$ is generated from a uniform distribution in $[-0.4 ; 0.4]$, for each OFDM symbol. Such CFO severely degrades the received signal and a perfect CPE correction 


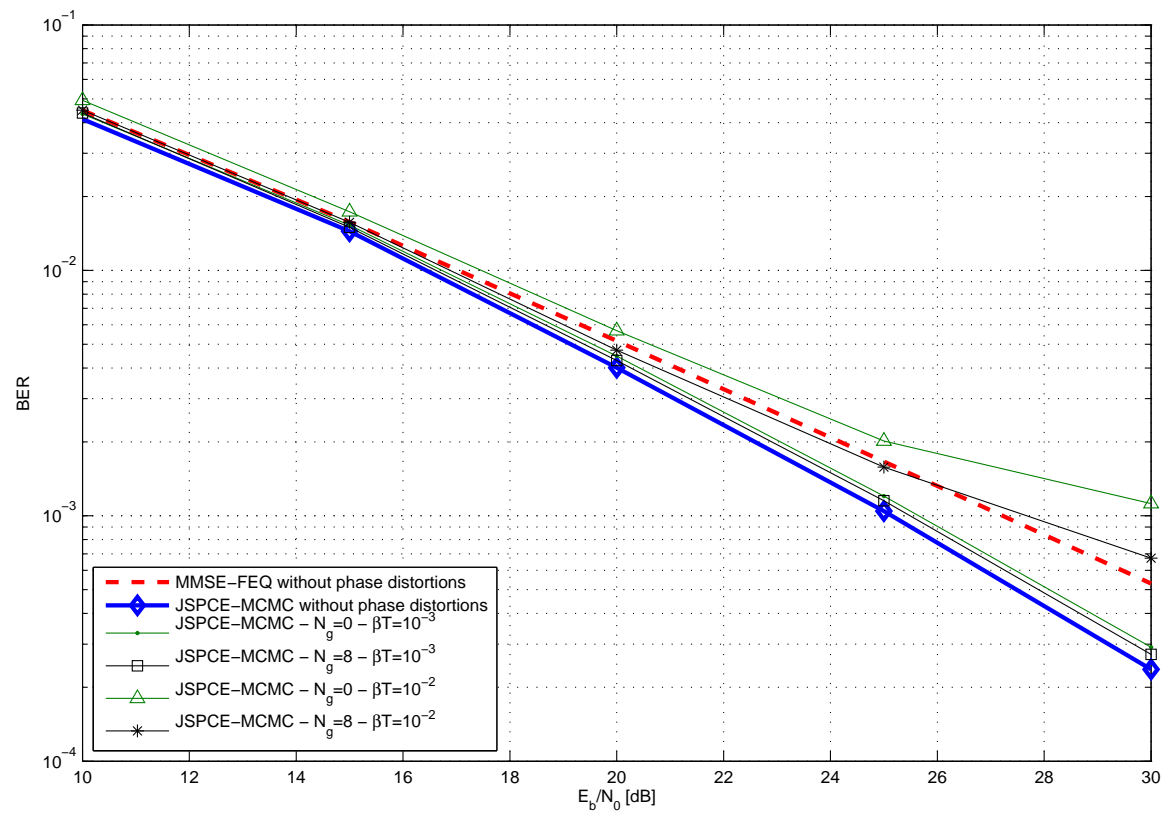

Fig. 3. BER performance of the proposed JSPCE-MCMC vs $E_{b} / N_{0}$ for different PHN rates $\beta T$ and number of null subcarriers $N_{g}$ in an OFDM system $(P=4, \epsilon=0)$.

obtain unsatisfactory BER performances due to a large ICI. In this context, the existing algorithms based on pilot subcarriers leads consequently to poor performances since a CPE correction scheme is required for the decision-directed method at the initialization step. Fig. 7 shows the BER performance of the proposed algorithm for different PHN rate and also different number of null-subcarriers. Firstly, only a slight performance degradation is observed when a severe CFO is considered in the OFDM system. Like in the CFO-free case, the BER performance and also the MSE of both the multicarrier signal and the phase distortions depicted in Figs. 8 and 9 are improved when the number of null subcarriers increases, owing to higher time correlation of the OFDM signal. These different results clearly highlight the efficiency of the proposed MCMC algorithm in this severe case.

\section{CONCLUSiON}

In this paper, we address the difficult problem of the OFDM reception that suffers from the presence of phase noise and carrier frequency offset. To solve this problem, we propose a novel time domain approach which has the great advantage of taking into account the redundancy information induced by 

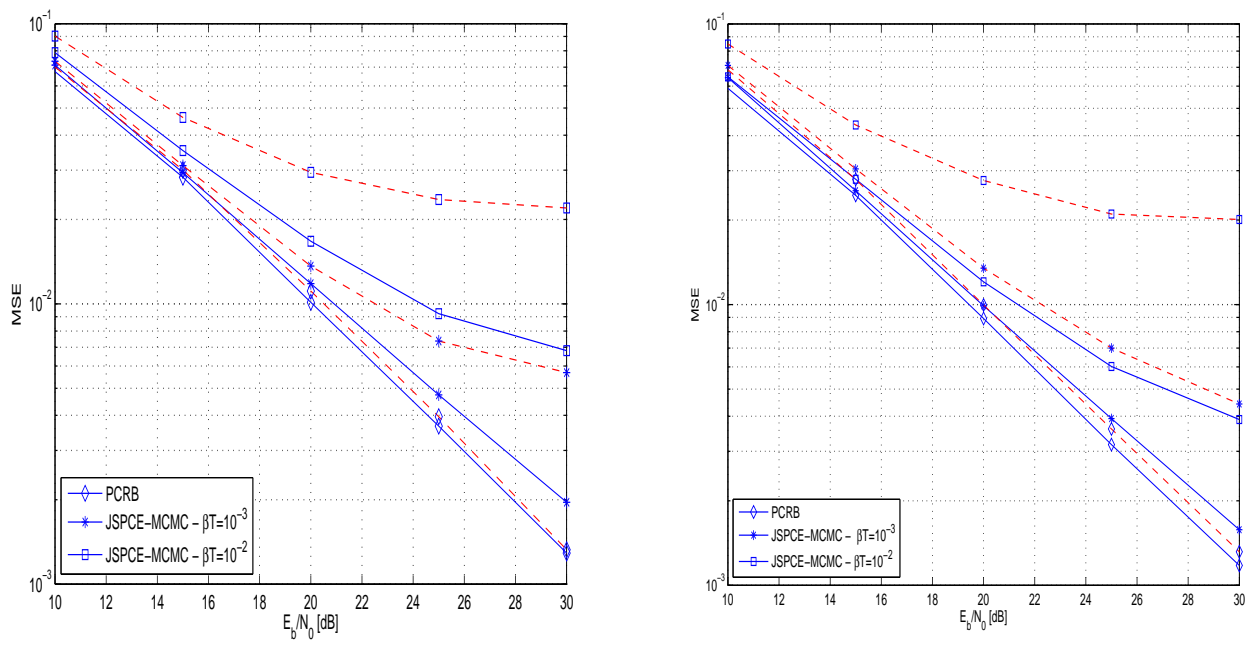

Fig. 4. MSE of the multicarrier signal estimate using the time correlation of the OFDM signal (blue solid lines) or not (red dashed lines) vs $E_{b} / N_{0}$ for different PHN rates $\beta T(P=4, \epsilon=0)$ with $N_{g}=0$ (left) and $N_{g}=8$ (right).
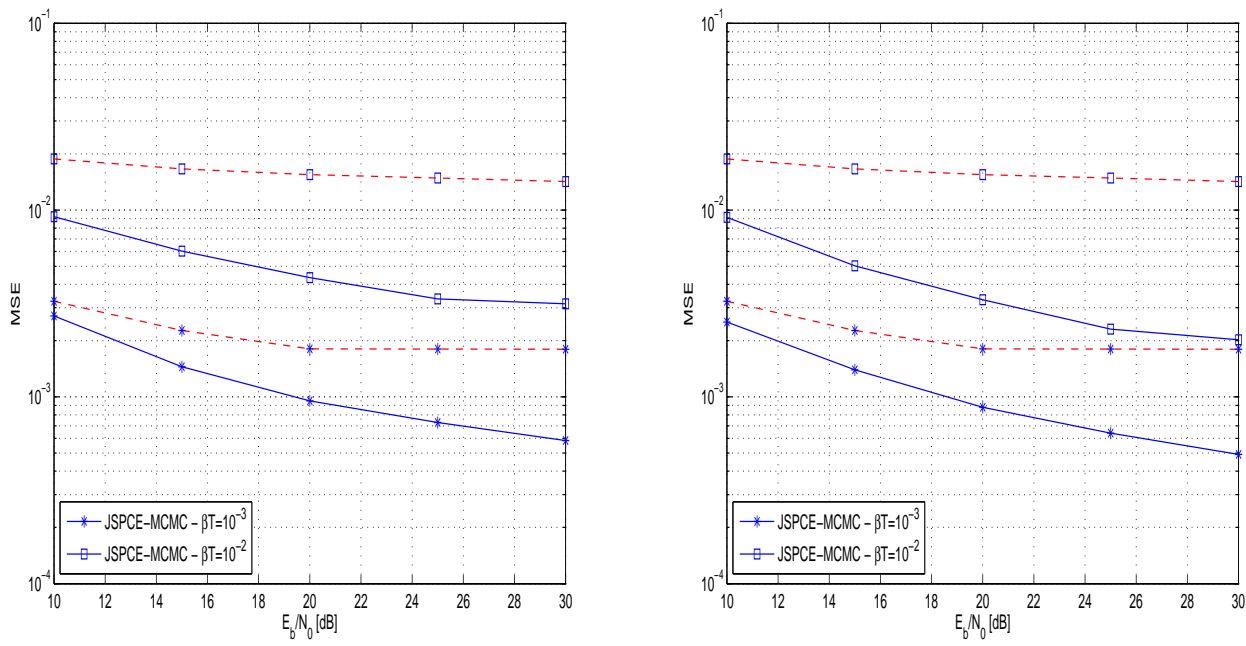

Fig. 5. MSE of phase distortion estimate using the time correlation of the OFDM signal (blue solid lines) or not (red dashed lines) vs $E_{b} / N_{0}$ for different PHN rates $\beta T(P=4, \epsilon=0)$ with $N_{g}=0$ (left) and $N_{g}=8$ (right).

the cyclic prefix and the time correlation of the OFDM signal owing to the presence of virtual and/or pilot subcarriers. A MCMC algorithm is proposed to approximate the posterior distribution of the high dimensional state of interest. The proposed algorithm is efficiently designed by incorporating the Rao- 


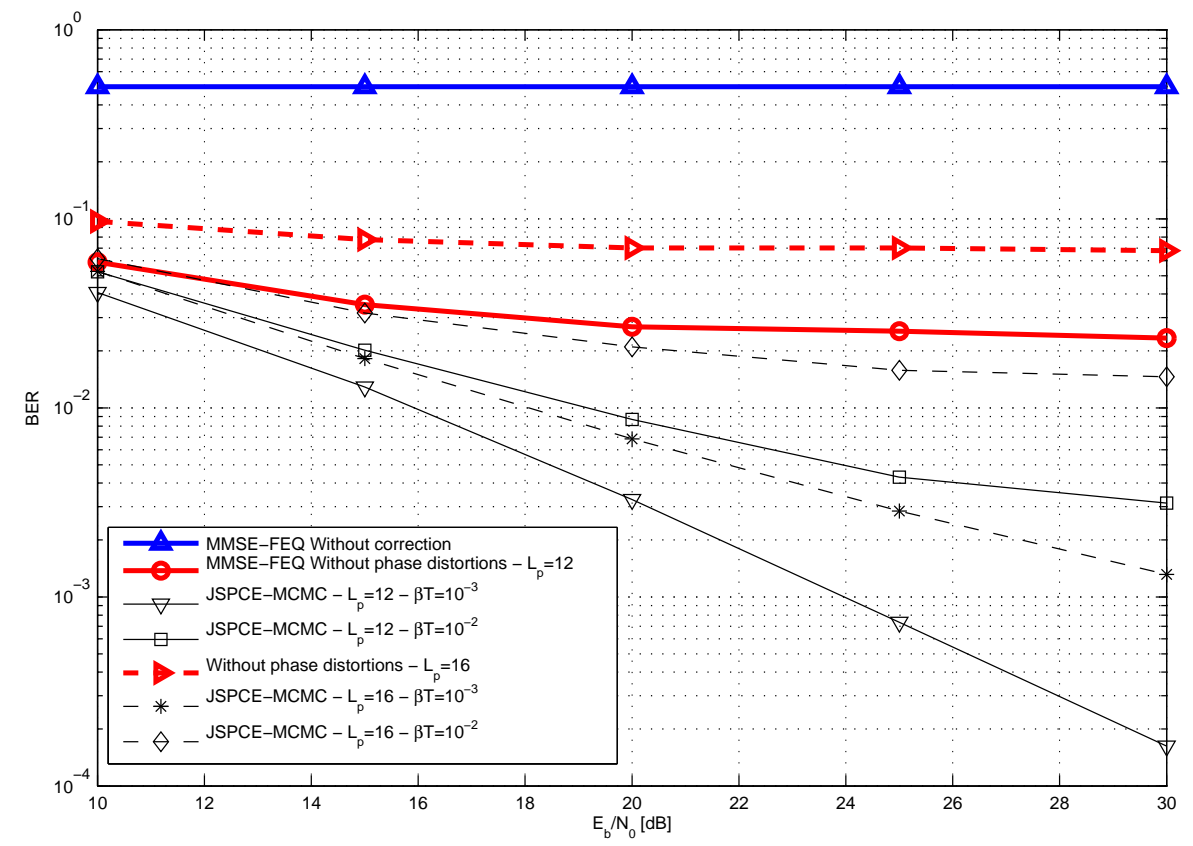

Fig. 6. BER performance of the proposed JSPCE-MCMC when the number of channel paths is superior to the cyclic prefix length, i.e $L_{p}>N_{c p}=8$, for different PHN rates $\beta T$ in an OFDM system $\left(N_{g}=0, P=4, \epsilon=0\right)$.

Blackwellization technique as well as optimized proposal distributions.

Numerical simulations show that even with significant PHN rates, the JSPCE-MCMC achieves good performances both in terms of the phase distortion estimation and BER. In particular, it was found that, for a small PHN rate, the JSPCE-MCMC is more efficient in term of BER than a frequency MMSE equalizer in multicarrier system without phase distortions. Moreover, the proposed JSPCE-MCMC enables to cope with a channel delay spread longer than one of the cyclic prefix. Thus, the JSPCE-MCMC algorithm offers a significant performance gain in comparison to existing methods and can be efficiently used with the channel estimator proposed in [20] for the design of a complete multicarrier receiver in wireline and wireless communication systems.

\section{APPENDIX A}

\section{DERIVATION OF THE PHN PROPOSAL DISTRIBUTION BASED ON OBSERVATIONS}

Let us firstly remark that the observation equation in (17) can be equivalently written as :

$$
\mathbf{r}_{n}=\boldsymbol{\Upsilon}_{n} \exp \left(j \boldsymbol{\theta}_{n}\right)+\mathbf{b}_{n}
$$




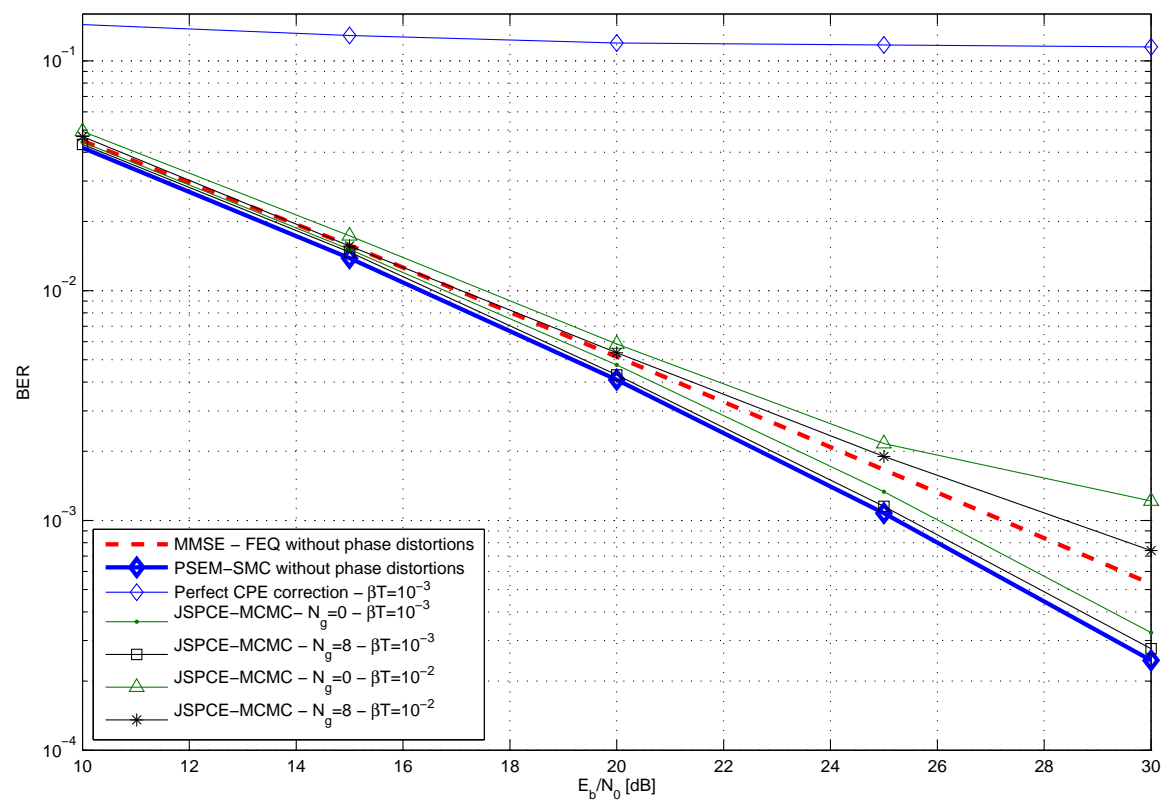

Fig. 7. BER performance of the proposed JSPCE-MCMC vs $E_{b} / N_{0}$ for different PHN rates $\beta T$ and number of null-subcarriers $(P=4, \epsilon=0.4)$.

where $\boldsymbol{\Upsilon}_{n}=\operatorname{diag}\left(\operatorname{diag}\left(e^{j \mathbf{g} \epsilon}\right)\left(\mathbf{H}_{n} \mathbf{A}\left(\boldsymbol{\kappa}_{n}+\mathbf{u}_{n}\right)+\boldsymbol{\nu}_{n}\right)\right)$. From this equation and given the current Markov chain state, $p\left(\mathbf{r}_{n} \mid \boldsymbol{\theta}_{n}, \epsilon^{m}, \mathbf{u}_{n}=\widehat{\mathbf{u}}_{n}^{m}\right)$ is given by :

$$
p\left(\mathbf{r}_{n} \mid \boldsymbol{\theta}_{n}, \epsilon^{m}, \mathbf{u}_{n}=\widehat{\mathbf{u}}_{n}^{m}\right)=\mathcal{N}_{c}\left(\mathbf{r}_{n} \mid \mathbf{\Upsilon}_{n}^{m} \exp \left(j \boldsymbol{\theta}_{n}\right), \sigma_{b}^{2} \mathbf{I}_{N+N_{c p}}\right)
$$

The vector $\widehat{\mathbf{u}}_{n}^{m}$ is obtained using Eq. (21) and the current Markov chain state $\left\{\epsilon^{m}, \boldsymbol{\theta}^{m}\right\}$. By using the block notation with the subscript $i$ as described in Section III-C and since $\Upsilon_{n}^{m}$ is diagonal, the following model can be considered to build the proposal distribution for $\boldsymbol{\theta}_{n, i}$ :

$$
\begin{gathered}
p\left(\boldsymbol{\theta}_{n, i} \mid \boldsymbol{\theta}_{n, \backslash i}^{m}\right)=\mathcal{N}\left(\boldsymbol{\theta}_{n, i} \mid \overline{\boldsymbol{\theta}}_{n, i}^{m}, \overline{\boldsymbol{\Theta}}_{n, i}\right) \\
p\left(\mathbf{r}_{n, i} \mid \boldsymbol{\theta}_{n, i}, \epsilon^{m}, \mathbf{u}_{n, i}=\widehat{\mathbf{u}}_{n, i}, \boldsymbol{\theta}_{n, \backslash i}^{m}\right)=\mathcal{N}_{c}\left(\mathbf{r}_{n, i} \mid \mathbf{\Upsilon}_{n, i}^{m} \exp \left(j \boldsymbol{\theta}_{n, i}\right), \sigma_{b}^{2} \mathbf{I}_{\frac{N+N_{c p}}{I}}\right)
\end{gathered}
$$

The conditional prior distribution is defined in (29). From this prior and likelihood information, we propose to use, as our proposal distribution for $\boldsymbol{\theta}_{n, i}$, the approximation of the posterior distribution obtained using the linearization of $\exp \left(j \boldsymbol{\theta}_{n, i}\right)$. As a consequence, we obtain :

$$
q_{1}\left(\boldsymbol{\theta}_{n, i} \mid \boldsymbol{\theta}_{n}^{m}, \epsilon^{m}, \mathbf{r}_{n}\right)=\mathcal{N}\left(\boldsymbol{\theta}_{n, i} \mid \widehat{\boldsymbol{\theta}}_{n, i}^{m}, \boldsymbol{\Sigma}_{\theta}^{m}\right)
$$




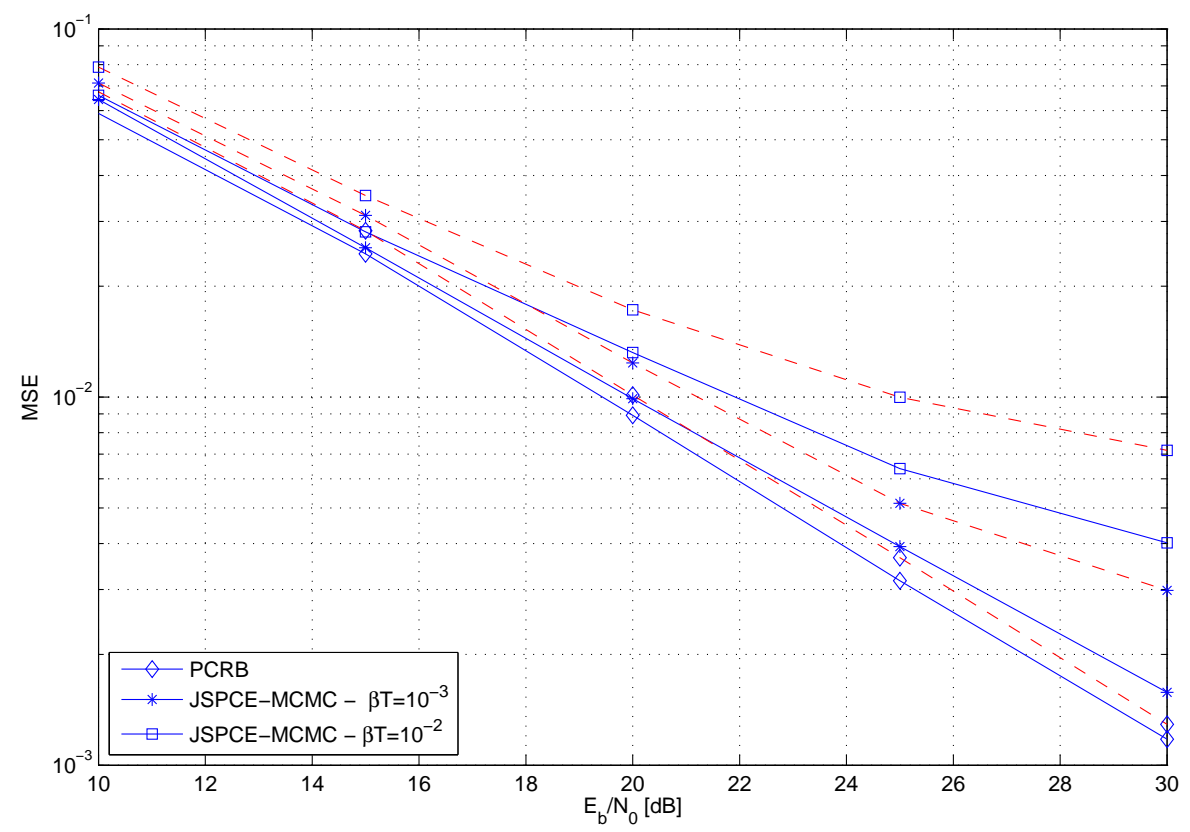

Fig. 8. MSE of the multicarrier signal estimate vs $E_{b} / N_{0}$ for different PHN rates $\beta T\left(N_{g}=0, P=4, \epsilon=0.4\right)$ with $N_{g}=0$ (red dashed lines) and $N_{g}=8$ (blue solid lines).

where the mean vector and the covariance matrix are given respectively by :

$$
\begin{aligned}
\widehat{\boldsymbol{\theta}}_{n, i}^{m} & =\overline{\boldsymbol{\theta}}_{n, i}^{m}+\mathbf{P}_{n, i} \widetilde{\mathbf{r}}_{n, i} \\
\boldsymbol{\Sigma}_{\theta}^{m} & =\left(\mathbf{I}_{\frac{N+N_{c p}}{I}}-j \mathbf{P}_{n, i} \mathbf{\Upsilon}_{n, i}^{m}\right) \overline{\mathbf{\Theta}}_{n, i}
\end{aligned}
$$

with

$$
\begin{aligned}
\widetilde{\mathbf{r}}_{n, i} & =\mathbf{r}_{n, i}-\mathbf{\Upsilon}_{n, i}^{m} \exp \left(j \overline{\boldsymbol{\theta}}_{n, i}^{m}\right) \\
\mathbf{S}_{n, i} & =j \mathbf{\Upsilon}_{n, i}^{m} \overline{\boldsymbol{\Theta}}_{n, i}\left(j \mathbf{\Upsilon}_{n, i}^{m}\right)^{H}+\sigma_{b}^{2} \mathbf{I}_{\frac{N+N_{c p}}{I}} \\
\mathbf{P}_{n, i} & =\overline{\boldsymbol{\Theta}}_{n, i}\left(j \mathbf{\Upsilon}_{n, i}^{m}\right)^{H} \mathbf{S}_{n, i}^{-1}
\end{aligned}
$$

\section{REFERENCES}

[1] P. Robertson and S. Kaiser, "Analysis of the effects of phase noise in orthogonal frequency division multiplexing (OFDM) systems," in Proc. IEEE ICC, 1995, pp. 1652-1657.

[2] D. Yee, J. Reilly, and T. Kirubarajan, "Channel equalization and phase noise suppression for OFDM systems in a timevarying frequency channel using particle filtering," in Proc. IEEE Workshop ICASSP, Mar. 2005. 


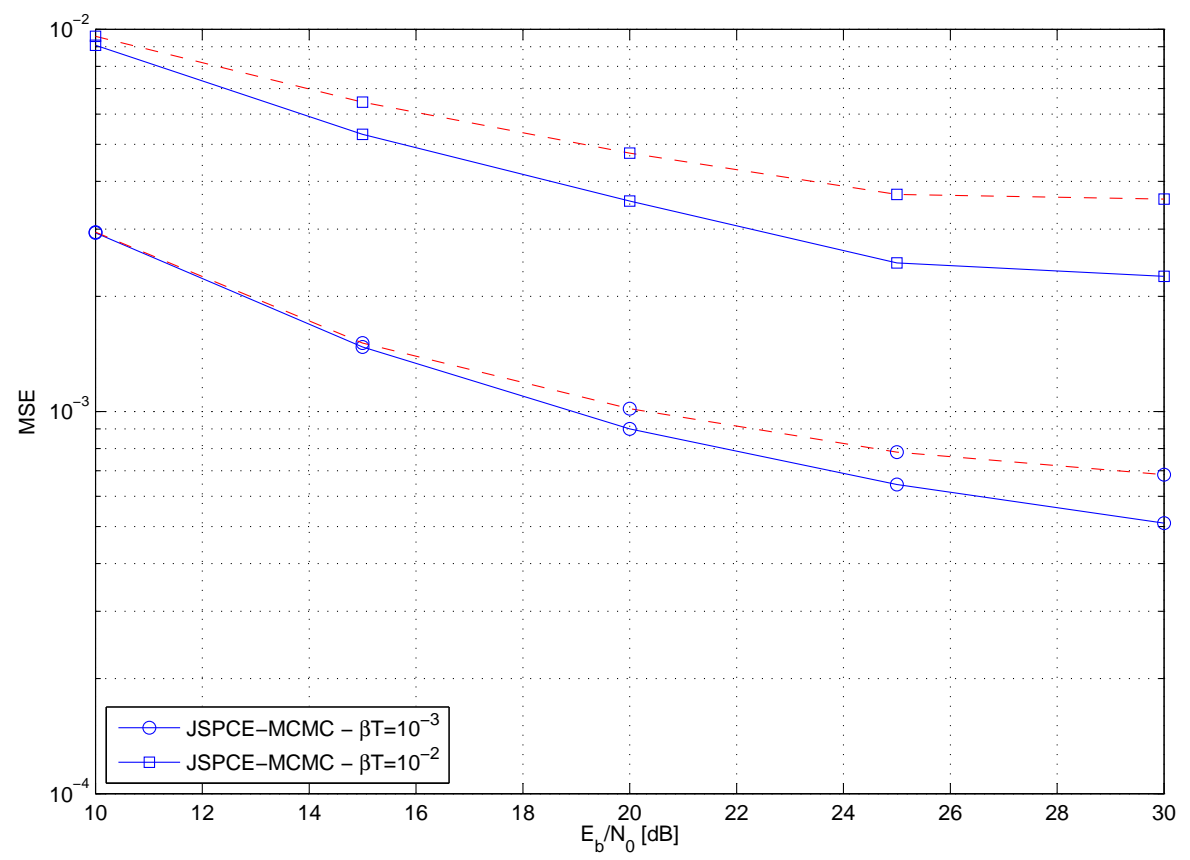

Fig. 9. MSE of phase distortion estimate vs $E_{b} / N_{0}$ for different PHN rates $\beta T\left(N_{g}=0, P=4, \epsilon=0.4\right)$ with $N_{g}=0$ (red dashed lines) and $N_{g}=8$ (blue solid lines).

[3] D. Petrovic, W. Rave, and G. Fettweis, "Common phase error due to phase noise in OFDM - estimation and suppression," in Proc. IEEE PIMRC, 2004.

[4] S. Wu and Y. Bar-Ness, "OFDM systems in the presence of phase noise : consequences and solutions," IEEE Trans. Commun., vol. 52, pp. 1988-1996, Nov. 2004.

[5] D. Petrovic, W. Rave, and G. Fettweis, "Intercarrier interference due to phase noise in OFDM - estimation and suppression," in Proc. IEEE VTC Fall, Sep. 2004.

[6] S. Wu and Y. Bar-Ness, "A new phase noise mitigation method in OFDM systems with simultaneous CPE and ICI correction," in Proc. MCSS, Sep. 2003.

[7] K. Nikitopoulos and A. Polydoros, "Phase-impairment effects and compensation algorithms for OFDM systems." IEEE Trans. Commun., vol. 53, no. 4, pp. 698-707, Apr. 2005.

[8] R. A. Casas, S. L. Biracree, and A. E. Youtz, "Time domain phase noise correction for OFDM signals," IEEE Trans. Broadcast., 2002.

[9] D. D. Lin, Y. Zhao, and T. J. Lim, “OFDM phase noise cancellation via approximate probabilistic inference," in IEEE WCNC, Mar. 2005, pp. 27-32.

[10] D. D. Lin and T. J. Lim, "The variational inference approach to joint data detection and phase noise estimation in OFDM," IEEE Transactions on Signal Processing, vol. 55, no. 5, pp. 1862-1874, May 2007.

[11] F. Septier, Y. Delignon, A. Menhaj-Rivenq, and C. Garnier, "Non-pilot-aided sequential monte carlo method to joint 
signal, phase noise, and frequency offset estimation in multicarrier systems monte Carlo Methods for Channel, Phase Noise, Frequency Offset Estimation with Unknwon Noise Variances in OFDM Systems.” EURASIP Journal on Advances in Signal Processing, 2008.

[12] M. H. Chen, Q. M. Shao, and J. G. Ibrahim, Eds., Monte Carlo Methods for Bayesian Computation. New York: Springer Verlag, 2001.

[13] S. Wei, D. L. Goeckel, and P. A. Kelly, "The complex enveloppe of a bandlimited OFDM signal converges weakly to a Gaussian random process: proof and application," http://www.ece.lsu.edu/swei.

[14] L. Tomba, "On the effect of Wiener phase noise in OFDM systems," IEEE Transactions on Communications, vol. 46, pp. 580-583, May 1998.

[15] W. Gilks, S. Richardson, and D. Spiegelhalter, Markov Chain Monte Carlo in Practice. Chapman and Hall/CRC, 1996.

[16] C. P. Robert and G. Casella, Monte Carlo Statistical Methods, 2nd ed. Springer, 2004.

[17] W. K. Hastings, "Monte Carlo sampling methods using Markov chains and their application,” Biometrika, 1970.

[18] S. Geman and D. Geman, "Stochastic relaxation, Gibbs distributions, and the Bayesian restoration of images," IEEE Transactions on Pattern Analysis and Machine Intelligence, vol. 6, pp. 721-741, 1984.

[19] T. Hrycak and G. Matz, "Low-complexity time-domain ICI equalization for OFDM communications over rapidly varying channels," in Asilomar Conference on Signals, Systems and Computers, 2006, pp. 1767-1771.

[20] F. Septier, Y. Delignon, A. Menhaj-Rivenq, and C. Garnier, "Monte Carlo Methods for Channel, Phase Noise, Frequency Offset Estimation with Unknwon Noise Variances in OFDM Systems.” IEEE Transactions on Signal Processing, 2008. 